\title{
Matching conditions in the quasicontinuum method: Removal of the error introduced at the interface between the coarse-grained and fully atomistic region
}

Shimokawa, T.; Mortensen, Jens Jørgen; Schiøtz, Jakob; Jacobsen, Karsten Wedel

Published in:

Physical Review B Condensed Matter

Link to article, DOI:

10.1103/PhysRevB.69.214104

Publication date:

2004

Document Version

Publisher's PDF, also known as Version of record

Link back to DTU Orbit

Citation (APA):

Shimokawa, T., Mortensen, J. J., Schiøtz, J., \& Jacobsen, K. W. (2004). Matching conditions in the quasicontinuum method: Removal of the error introduced at the interface between the coarse-grained and fully atomistic region. Physical Review B Condensed Matter, 69(21), 214104.

https://doi.org/10.1103/PhysRevB.69.214104

\section{General rights}

Copyright and moral rights for the publications made accessible in the public portal are retained by the authors and/or other copyright owners and it is a condition of accessing publications that users recognise and abide by the legal requirements associated with these rights.

- Users may download and print one copy of any publication from the public portal for the purpose of private study or research.

- You may not further distribute the material or use it for any profit-making activity or commercial gain

- You may freely distribute the URL identifying the publication in the public portal 


\title{
Matching conditions in the quasicontinuum method: Removal of the error introduced at the interface between the coarse-grained and fully atomistic region
}

\author{
T. Shimokawa, ${ }^{1,2,3}$ J. J. Mortensen, ${ }^{3}$ J. Schiøtz, ${ }^{3}$ and K. W. Jacobsen ${ }^{3}$ \\ ${ }^{1}$ Department of Mechanical Systems Engineering, Kanazawa University, 2-40-20 Kodatsuno, Kanazawa, Ishikawa 920-8667, Japan \\ ${ }^{2}$ Department of Adaptive Machine Systems, Graduate School of Engineering, Osaka University, 2-1 Yamadaoka, Suita, \\ Osaka 565-0871, Japan \\ ${ }^{3}$ CAMP and Department of Physics, Technical University of Denmark, DK-2800 Lyngby, Denmark \\ (Received 4 September 2003; revised manuscript received 23 January 2004; published 15 June 2004)
}

\begin{abstract}
The quasicontinuum method is a way of reducing the number of degrees of freedom in an atomistic simulation by removing the majority of the atoms in regions of slowly varying strain fields. Due to the different ways the energy of the atoms is calculated in the coarse-grained regions and the regions where all the atoms are present, unphysical forces called "ghost forces" arise at the interfaces. Corrections may be used to almost remove the ghost forces, but the correction forces are nonconservative, ruining energy conservation in dynamic simulations. We show that it is possible to formulate the quasicontinuum method without these problems by introducing a buffer layer between the two regions of space. The method is applicable to short-ranged potentials in the face-centered cubic, body-centered cubic, and hexagonal close-packed crystal structures.
\end{abstract}

DOI: 10.1103/PhysRevB.69.214104

PACS number(s): 62.20.-x, 02.70.Ns, 46.15.-x

\section{INTRODUCTION}

A successful description of material properties sometimes involves a careful description of the material at both atomic and microscopic length scales. A couple of methods have been proposed for coupling these length scales, including the coupled finite element/molecular-dynamics/tight-binding method, ${ }^{1,2}$ the coarse-grained molecular-dynamics method, ${ }^{3,4}$ and the quasicontinuum (QC) method, ${ }^{5-7}$ the latter being the subject of this paper.

The QC method combines continuum and atomic descriptions, thus allowing for an efficient description of threedimensional (3D) systems where several length scales are of importance ${ }^{8-11}$ A semiempirical interatomic potential is used for the atomistic region and the continuum region is described by a special finite element method, where the elements are tetrahedra with atoms at the corners. Each element has a crystal orientation matching the corner atoms. The element region is joined to the atomistic region giving rise to an interface between the two regions.

It turns out to be very difficult to define the energy of the interface region in such a way that the coupling of the two descriptions is completely seamless. By seamless, we mean no discontinuities in the force, stress, and displacement fields. We have found that by introducing a buffer layer of atoms, to be treated in a special way, between the element region and the atomistic region, we can achieve a seamless coupling of the two descriptions.

In the following we will first describe the traditional QC method and how it fails at interfaces. After introducing the concept of local and nonlocal atoms from Ref. 6, we develop the idea of a different kind of quasi-nonlocal atom, which will enable us to seamlessly couple local and nonlocal regions, and account for how to calculate the energy of quasinonlocal atoms. The method is limited to potentials with a relatively short range. In face-centered-cubic (fcc) and hexagonal-close-packed (hcp) crystal structures, the range of the potential is limited to the fourth-nearest neighbors, and in body-centered-cubic (bcc) crystals to the third-nearest neighbors.

Finally, we will look at two illustrations of the new QC method: Calculation of the vacancy formation energy in $\mathrm{Al}$ and $\alpha$ iron, and a calculation of the grain boundary energy of a tilt boundary in $\mathrm{Cu}$. The $\mathrm{Al}$ calculations use an embedded atom model (EAM) potential, ${ }^{12}$ the $\alpha$-iron calculations use a Finnis-Sinclair $N$-body potential, ${ }^{13,14}$ and for the $\mathrm{Cu}$ calculations we use an effective medium theory (EMT) potential. ${ }^{15}$ The cutoff distance is between fourth- and fifth-nearest neighbors for the EAM, between second- and third-nearest neighbors for the $N$-body potential, and between third and fourth for the EMT potential.

\section{THE QUASICONTINUUM METHOD}

The basic idea of the QC method is very simple. In order to perform an atomic simulation efficiently and save computational resources, a continuum approximation is adopted where atomic deformation-gradient fields are small, assuming that the continuum method provides almost the same result as a full atomistic simulation. Figure 1(a) shows a full atomistic configuration near a vacancy (model $A$ ). The energy of the system is given by a sum of atomic energies

$$
E^{A}=\sum_{\alpha \in N^{A}} E_{\alpha}^{N}
$$

where $E_{\alpha}^{N}$ is the energy of atom $\alpha$ and $N^{A}$ is the set of all atoms in system $A$. We call these atoms nonlocal ${ }^{6}$ because the energy depends on the positions of all atoms within a certain cutoff distance $R_{c}$ from the atoms. The force acting on atom $\alpha$ is then 


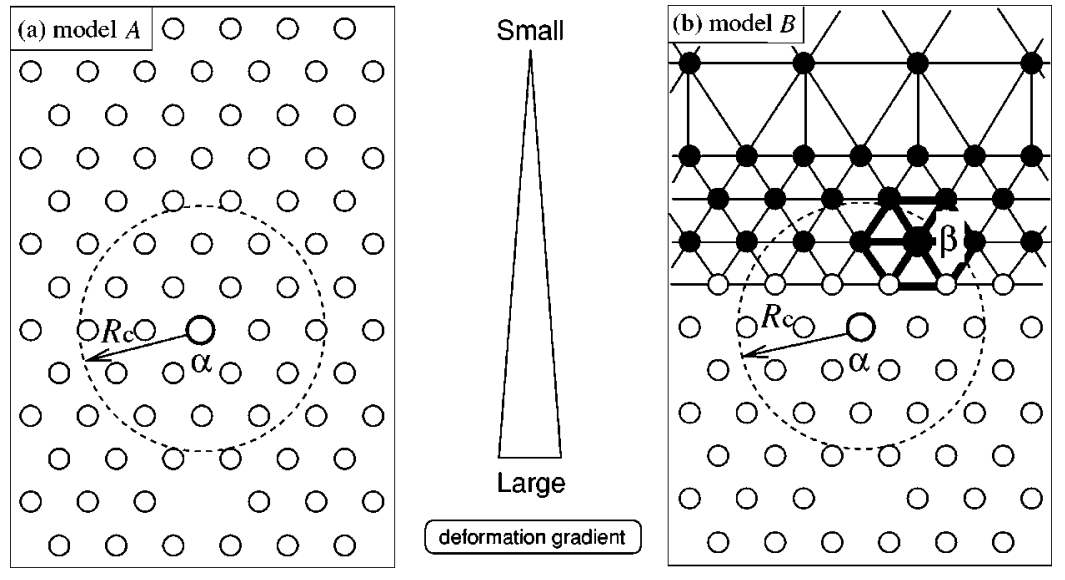

FIG. 1. Coupling of atomic and continuum model with one vacancy. To the left is a fully atomistic model, to the right a quasicontinuum model. In the quasicontinuum model open circles show nonlocal atoms and solid circles show local atoms. In the small gradient fields far from the vacancy, each local atom in the quasicontinuum model represents several nonlocal atoms in the fully atomistic model.

$$
\mathbf{F}_{\alpha}^{A}=-\frac{\partial E_{\alpha}^{N}}{\partial \mathbf{r}_{\alpha}}-\sum_{\alpha^{\prime} \in N_{\alpha}^{A}} \frac{\partial E_{\alpha^{\prime}}^{N}}{\partial \mathbf{r}_{\alpha}}
$$

where $N_{\alpha}^{A}$ is the set of (nonlocal) neighbors of atom $\alpha$ within the cutoff.

Figure 1(b) shows the QC model (model $B$ ) corresponding to model $A$. Here, open circles are nonlocal atoms treated exactly as in model $A$ and solid circles are so-called local atoms making up the corners of a triangulation of the region with a slowly varying deformation gradient. In this study the Delaunay triangulation ${ }^{16}$ is used to divide a system region into finite elements: in the two-dimensional (2D) example in Fig. 2(a), the elements of the triangulation are triangles and in the 3D case, the elements will be tetrahedra. Each local atom $\beta$ (corner atom) represents a certain number of atoms $\omega_{\beta}^{e}$ in each of the neighboring elements $e$. We define the energy of a local atom $\beta$ as

$$
E_{\beta}^{L}=\sum_{e} \omega_{\beta}^{e} E_{e}^{E}
$$

where $E_{e}^{E}$ is the energy of a nonlocal atom in an infinite crystal in a state of strain determined by the corners of the element $e$. (a)

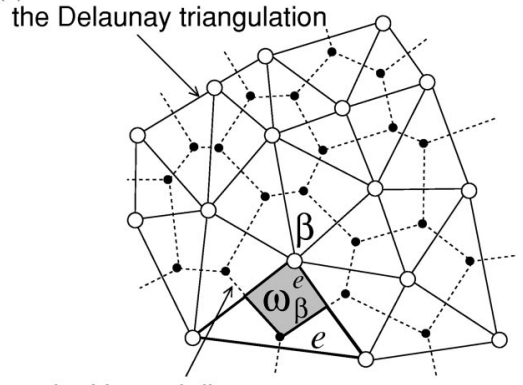

the Voronoi diagram (b)

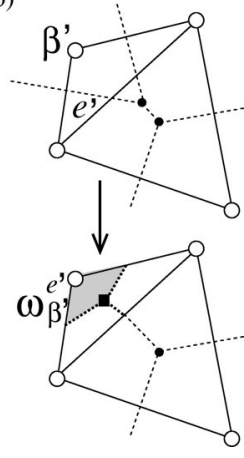

FIG. 2. Representative region $\omega_{\beta}^{e}$ of local atom $\beta$ in element $e$ is obtained by using the relation between the Delaunay triangulation (solid lines) and the Voronoi diagram (broken lines). When a corner of the Voronoi polyhedron of the element $e^{\prime}$ is outside the element, the center of gravity (solid square) is used to define $\omega_{\beta^{\prime}}^{e^{\prime}}$.
In order to calculate $E_{e}^{E}$ in the $3 \mathrm{D}$ case, each element $e$ must have a local set of lattice basis vectors $\mathbf{a}_{e}^{1}, \mathbf{a}_{e}^{2}$, and $\mathbf{a}_{e}^{3}$, describing the strain state of the infinite crystal. The lattice basis vectors of an element are linearly related to the positions of the four corner atoms (see, e.g., Ref. 11). Everything outside an element is irrelevant for its energy - that is where the term local comes from.

We have to divide an element $e$ into representative regions for the corner atoms $\beta$ to decide the values of $\omega_{\beta}^{e}$. The representative region of each local atom can be defined by using the Voronoi diagram. This is the dual structure of the Delaunay triangulation, so one corner of the Voronoi polyhedron is certain to correspond to one element. Hence, $\omega_{\beta}^{e}$ can be obtained as the size of the region of the element $e$ which is part of the Voronoi polyhedron of the atom $\beta$. Notice that in a case of an element with a narrow shape as shown in Fig. 2(b), a corner of the Voronoi polyhedron is outside of the corresponding element, so the element region cannot be divided by the Voronoi diagram. In this case, the element region is divided by the position of its center of gravity. As an alternative to using the Voronoi diagram, one could use the solid angle of the corner of the element to determine $\omega_{\beta}^{e}$. The values of $\omega_{\beta}^{e}$ are determined at the beginning of the simulation, and remain fixed during the simulation.

Let $N^{B}$ and $L^{B}$ be the nonlocal and local atoms, respectively, of system $B$. For the QC system $B$, the total energy is

$$
E^{B}=\sum_{\alpha \in N^{B}} E_{\alpha}^{N}+\sum_{\beta \in L^{B}} E_{\beta}^{L} .
$$

To compare with system $A$, we now write down the force acting on atom $\alpha$ in the model $B$

$$
\mathbf{F}_{\alpha}^{B}=-\frac{\partial E_{\alpha}^{N}}{\partial \mathbf{r}_{\alpha}}-\sum_{\alpha^{\prime} \in N_{\alpha}^{B}} \frac{\partial E_{\alpha^{\prime}}^{N}}{\partial \mathbf{r}_{\alpha}}-\sum_{\beta \in L^{B}} \frac{\partial E_{\beta}^{L}}{\partial \mathbf{r}_{\alpha}},
$$

where $N_{\alpha}^{B}$ is the nonlocal neighbors of atom $\alpha$ within the cutoff $R_{c}$ for model $B$.

Looking at the specific nonlocal atom $\alpha$ depicted in Figs. 1(a) and 1(b), we now compare $\mathbf{F}_{\alpha}^{A}$ and $\mathbf{F}_{\alpha}^{B}$, which ideally should be equal. The $\alpha$ atom in the model $B$ is not a corner of any element, so the last terms in Eq. (5) vanishes. Subtracting, we get for $\mathbf{F}_{\alpha}^{B}-\mathbf{F}_{\alpha}^{A}$ 


$$
\sum_{\alpha^{\prime} \in N_{\alpha}^{A} \backslash N_{\alpha}^{B}} \frac{\partial E_{\alpha^{\prime}}^{N}}{\partial \mathbf{r}_{\alpha}},
$$

where $N_{\alpha}^{A} \backslash N_{\alpha}^{B}$ is the set of nonlocal neighbors of the $\alpha$ atom present in the model $A$ and not in the model $B$-in other words: The neighbor atoms of atom $\alpha$ that have been changed from nonlocal atoms in model $A$ to local atoms in model $B$.

The difference in Eq. (6) is nonzero for a nonlocal atom close to a nonlocal/local interface, which proves the existence of unphysical forces (ghost forces) in the simple QC model. Similarly, one can show that there are ghost forces acting on local atoms close to a nonlocal/local interface. These ghost forces will be present even if the atoms have the positions of a perfect crystal. Relaxing the structure of model $B$ to remove the forces in the perfect crystal structure, will lead to an unphysical ground-state energy lower than that of the correct model $A$.

One way to remove the ghost forces is to introduce "static" correction forces as it is done in Ref. 6. Relaxing the structure using these forces will require a recalculation of the correction forces, since they depend on the atomic positions. The updating of the correction forces can be done more or less frequently. ${ }^{6}$ A very serious problem with the use of correction forces is that they are not derivable from a "correction potential energy," i.e., they are nonconservative. We have found that this leads to serious problems with energy conservation during a molecular-dynamics simulation. Other ways of handling the ghost force problem also result in nonconservative forces. ${ }^{11} \mathrm{~A}$ more elegant solution to the ghost force problem would clearly be desirable. In this study, we improve the QC method so that atomic forces derived from the total energy are well behaved: No ghost forces and a seamless interface between local and nonlocal regions.

Knap and $\mathrm{Ortiz}^{8}$ approach the problem in an alternative way, by changing the description of the coarse-grained region. Instead of calculating the energy of each homogeneously strained element, they use small clusters of atoms around each corner atom. This avoids the ghost forces, but introduces an approximation to the energy and forces in the local region. This approximation can be improved by increasing the cluster size ${ }^{8}$ at the expense of an increase in the computational burden.

\section{A SEAMLESS COUPLING OF ATOMISTIC AND CONTINUUM REGIONS}

The problem with ghost forces is due to the fact that the interaction range of a nonlocal atom is different from that of a local atom. To solve this problem, we introduce a type of atom, named quasi-nonlocal atom, to be positioned between the nonlocal and local regions. The concept of a quasinonlocal atom is very simple: A quasi-nonlocal atom can feel first nearest-neighbor atoms and all nonlocal atoms within the cutoff distance $R_{c}$. The idea of the improved QC model is shown in Fig. 3(a) (model $C$ ). Double circles correspond to quasi-nonlocal atoms. Quasi-nonlocal atoms are located as a buffer in between local and nonlocal atoms, so that no nonlocal atom interacts with any local atom.

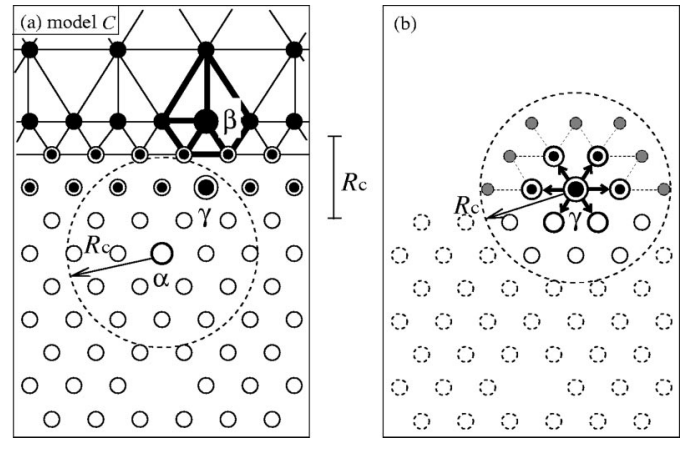

FIG. 3. Concept of a quasi-nonlocal atom. (a) Quasi-nonlocal atoms (double circles) are located between nonlocal and local atomic regions. (b) The quasi-nonlocal atom $\gamma$ images neighbor atoms (gray circles) by using first neighbor atomic positions (big open and double circles) except for nonlocal atoms (small open circles) within the interactive field.

The potential energy of a quasi-nonlocal atom is calculated as if it was a nonlocal atom with one important difference: When calculating the energy of a quasi-nonlocal atom, only the positions of nearest-neighbor atoms as well as the positions of nonlocal atoms within the cutoff distance are used. On the local side of the interface, the distance vectors to the nearest-neighbor atoms are used to extrapolate the positions of second nearest neighbors, third nearest neighbors, and so on. This is illustrated in Fig. 3(b). Big open circles and double circles around the quasi-nonlocal atom $\gamma$ are the nearest-neighbor atoms, gray circles are extrapolated neighbor atoms, and broken circles are nonlocal atoms not needed to calculate the potential energy of the quasi-nonlocal atom $\gamma$. If the position of an extrapolated neighbor atom and the positions of a nonlocal atom are almost the same, the nonlocal atom is used instead of the extrapolated position. ${ }^{17} \mathrm{By}$ using these extrapolated neighbor atoms, we have enough information to calculate the potential energy of a quasinonlocal atom in the same way as for a nonlocal atom. Note that local atoms near the interface must be located at all lattice sites, because quasi-nonlocal atoms need first neighbor atoms for extrapolation of atoms further away than nearest-neighbor atoms. As a result, each quasi-nonlocal atom represents only one atom-just as a nonlocal atom. Consequently, a quasi-nonlocal atom acts like a nonlocal atom on the nonlocal side of the interface, and it acts like a local atom on the local side of the interface.

The total energy of the improved QC model is given by

$$
E=\sum_{\alpha \in N} E_{\alpha}^{N}+\sum_{\gamma \in Q} E_{\gamma}^{Q}+\sum_{\beta \in L} E_{\beta}^{L},
$$

where $N, Q$, and $L$ are the sets of nonlocal, quasi-nonlocal, and local atoms respectively.

As an example, let us look at the two-dimensional hexagonal lattice shown in Fig. 4(a). The quasi-nonlocal atom $\gamma^{1}$ extrapolates the second-nearest-neighbor atom at position 14 by using $\gamma^{5}$ and $\gamma^{6}$, and $\gamma^{14}$ extrapolates the neighbor atom at 1 by using the same $\gamma^{5}$ and $\gamma^{6}$, so these quasi-nonlocal atoms extrapolate the neighbor atom corresponding to each quasinonlocal atomic position by using common first neighbor 


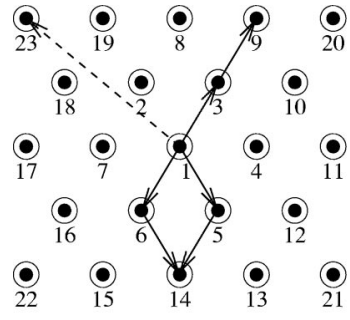

(a)

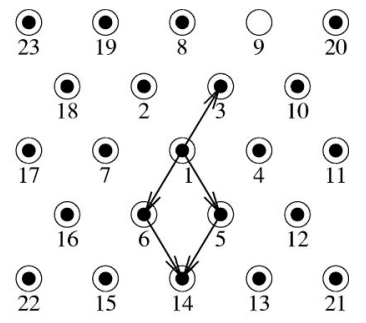

(b)
FIG. 4. (a) In a 2D hexagonal lattice, the quasi-nonlocal atom 1 can extrapolate the positions of its next-nearest neighbors (such as atom 14) and its third-nearest neighbors (such as atom 9) from common nearest neighbors (atoms $5+6$ and atom 3, respectively). But it cannot extrapolate the position of fourth-nearest neighbors (such as atom 23) from common nearest neighbors. (b) If a neighbor such as atom 9 is a nonlocal atom, its position is not extrapolated.

atoms. Similarly, the quasi-nonlocal atom $\gamma^{1}$ extrapolates the third-nearest-neighbor atom at position 9 by using $\gamma^{3}$, and $\gamma^{9}$ will extrapolate the position of its third-nearest-neighbor atom at position 1 by using the same $\gamma^{3}$. We see that the symmetry of interactions is restored. If we want to extrapolate the position of fourth-nearest neighbors, then we lose this symmetry: The atom at position 23 is a fourth-nearest neighbor of $\gamma^{1}$ and it can be extrapolated by using $\gamma^{2}$ and $\gamma^{7}$, but $\gamma^{23}$ will use $\gamma^{18}$ and $\gamma^{19}$ for extrapolation of atom $\gamma^{1}$. This gives an imbalance in the calculation of forces, resulting in ghost forces: for example, a displacement of atom $\gamma^{7}$ would result in atom $\gamma^{1}$ seeing a displacement of atom $\gamma^{23}$, but atom $\gamma^{23}$ would not see atom $\gamma^{1}$ moving, as atom $\gamma^{23}$ does not use atom $\gamma^{7}$ to calculate the position of atom $\gamma^{1}$. The conclusion is that we can extrapolate only the positions of atoms that are first-nearest neighbors to a first-nearest neighbor. This means that the range of the interatomic potential used for calculation of a two-dimensional hexagonal structure must be limited to include up to third-nearest neighbors only. If one of the neighboring atoms, for example, the atom at position 9 as shown in Fig. 4(b), is a nonlocal atom then the two atoms, $\gamma^{1}$ and $\alpha^{9}$, interact directly. Thus no asymmetry is introduced, and no ghost forces appear. This is shown in more detail in the Appendix.

We will now look at some particular crystal structures, and show how the extrapolation can be done. Using the example in Fig. 5, we want to write down expressions for a second-, third-, and fourth-nearest neighbor, using only the position of the central quasi-nonlocal atom $\gamma$ and the vectors pointing from the central atom to the nearest neighbors $\left(\mathbf{d}_{\eta}\right.$ $\left.=\mathbf{r}_{\eta}-\mathbf{r}_{\gamma}\right)$.

\section{A. Face-centered cubic}

Figure 5(a) shows first neighbors of the quasi-nonlocal atom $\gamma$ and the superposed atomic configurations on the (111) atomic planes. Full circles express nearest neighbors to the black atom $\gamma$ and middle, large, and small circles mean atoms in the same plane, one plane above or below as the black atom $\gamma$. Dashed circles correspond to first neighbors to the first neighbor (a) of the atom $\gamma$.

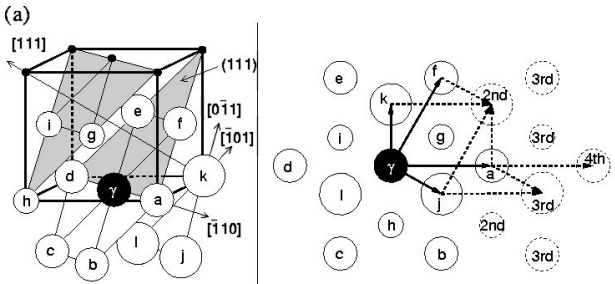

(b)
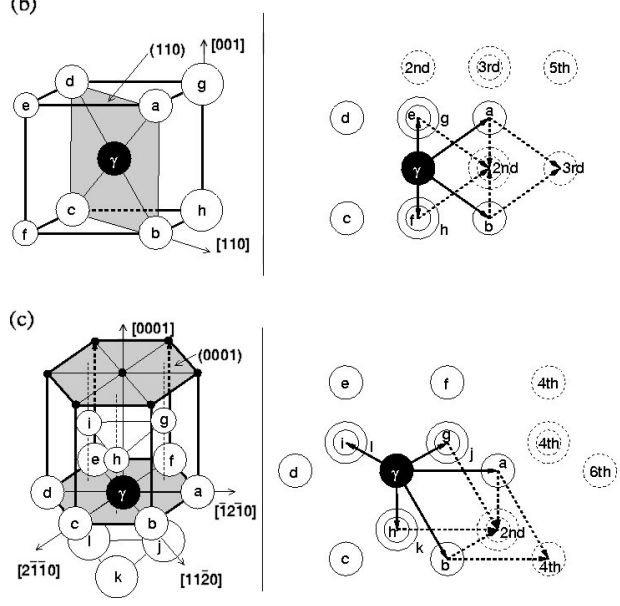

FIG. 5. First-nearest neighbors to the black atom $\gamma$ and superposed atomic configurations on (111), (110), and (0001) planes for (a) fcc, (b) bcc, and (c) hcp structures.

We can extrapolate neighbors by using the 12 difference vectors $\mathbf{d}_{\eta}$ as follows:

$$
\begin{gathered}
\mathbf{r}_{2 \mathrm{nd}}=\mathbf{r}_{\gamma}+\frac{1}{2}\left(\mathbf{d}_{\mathrm{a}}+\mathbf{d}_{\mathrm{f}}+\mathbf{d}_{\mathrm{j}}+\mathbf{d}_{\mathrm{k}}\right), \\
\mathbf{r}_{3 \mathrm{rd}}=\mathbf{r}_{\gamma}+\mathbf{d}_{\mathrm{a}}+\mathbf{d}_{\mathrm{j}}, \\
\mathbf{r}_{4 \mathrm{th}}=\mathbf{r}_{\gamma}+2 \mathbf{d}_{\mathrm{a}} .
\end{gathered}
$$

These three equations are chosen to be as symmetric as possible. In similar ways the other neighbors are found, for a total of 6 second-nearest, 24 third-nearest, and 12 fourthnearest neighbors.

A fifth-nearest neighbor is not a first-nearest neighbor of a first-nearest neighbor in the fcc structure, and the cutoff for the interatomic potential can therefore not include the fifthnearest neighbor shell.

\section{B. Body-centered cubic}

Figure 5(b) shows first neighbors to the quasi-nonlocal atom $\gamma$ and the superposed atomic configurations on the (110) atomic planes in the bcc structure. Using eight vectors $\mathbf{d}_{\eta}$, the positions of the neighbor atoms can be extrapolated as follows:

$$
\begin{gathered}
\mathbf{r}_{2 \text { nd }}=\mathbf{r}_{\gamma}+\frac{1}{2}\left(\mathbf{d}_{\mathrm{a}}+\mathbf{d}_{\mathrm{b}}+\mathbf{d}_{\mathrm{g}}+\mathbf{d}_{\mathrm{h}}\right), \\
\mathbf{r}_{3 \mathrm{rd}}=\mathbf{r}_{\gamma}+\mathbf{d}_{\mathrm{a}}+\mathbf{d}_{\mathrm{b}} .
\end{gathered}
$$

There are 6 and 12 equations for second and third neighbors, respectively. A fifth neighbor is a first neighbor of a 

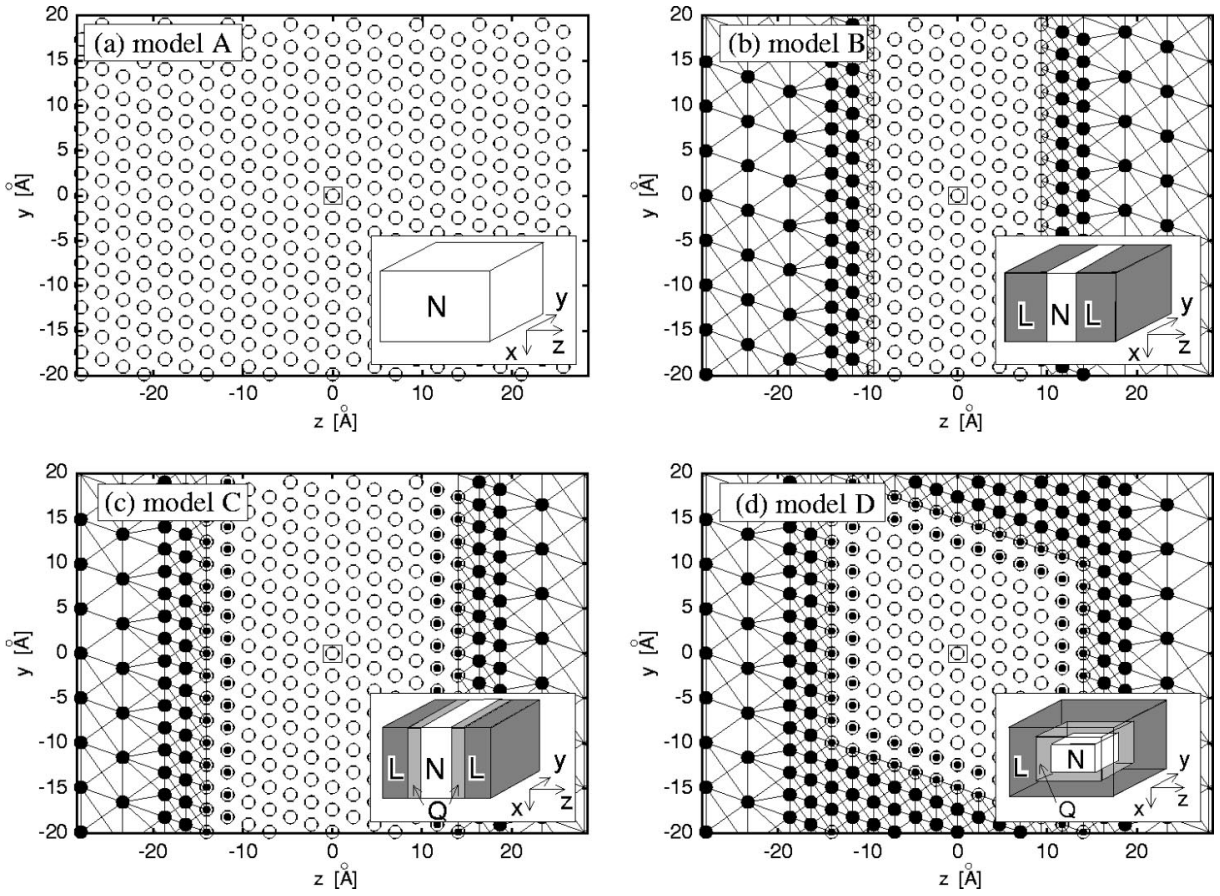

FIG. 6. Atomic configuration of each model. (a) Full atomistic model, (b) old style QC model without correction of ghost forces, (c) and (d) improved QC model with new buffer layers. Open, solid, and double circles represent nonlocal, local, and quasinonlocal atoms, respectively. first neighbor to the atom $\gamma$, but a fourth neighbor does not satisfy the extrapolation rule, so the cutoff for the interatomic potential cannot include the fourth-nearest-neighbor shell.

\section{Hexagonal closed packed}

The extrapolation rule will work with the hexagonalclose-packed lattice, if the potential is limited to fourthnearest neighbors. Figure 5(c) shows first-nearest neighbors to the atom $\gamma$ and the superposed atomic configurations on the (0001) atomic planes. A third neighbor is two layers directly above or below the black atom $\gamma$. The positions of the neighbors can be obtained by using 12 difference vectors $\mathbf{d}_{\eta}$ :

$$
\begin{gathered}
\mathbf{r}_{2 \mathrm{nd}}=\mathbf{r}_{\gamma}+\frac{1}{2}\left(\mathbf{d}_{\mathrm{a}}+\mathbf{d}_{\mathrm{b}}+\mathbf{d}_{\mathrm{g}}+\mathbf{d}_{\mathrm{h}}\right), \\
\mathbf{r}_{3 \mathrm{rd}}=\mathbf{r}_{\gamma}+\frac{2}{3}\left(\mathbf{d}_{\mathrm{g}}+\mathbf{d}_{\mathrm{h}}+\mathbf{d}_{\mathrm{i}}\right), \\
\mathbf{r}_{4 \mathrm{th}}=\mathbf{r}_{\gamma}+\mathbf{d}_{\mathrm{a}}+\mathbf{d}_{\mathrm{b}} .
\end{gathered}
$$

There are 6, 2, and 18 equations for second, third, and fourth neighbors, respectively.

\section{Other structures}

In a simple-cubic lattice, the extrapolation is only possible to second-nearest neighbors. This thus limits the range of the potentials for this crystal structure. Crystal structures which can be seen as linear distortions of fcc and bcc should clearly also work. This includes body-centered tetragonal unit cells, if the $c / a$ ratio is such that the crystal is not too far from a fcc or bcc lattice.

The formulation given here does not work for the diamond structure. An atom has 4 nearest neighbors, and 12 second-nearest neighbors, but a second-nearest neighbor only shares a single-nearest-neighbor atom with the central atom, making it impossible to extrapolate the position of a second-nearest neighbor from the positions of common nearest neighbors. This limits the applicability of the method to nearest-neighbor potentials only. However, with a nearestneighbor potential there are no ghost forces even in the original formulation of the quasicontinuum method, provided that a single fully resolved layer of local atoms is provided at the interface layer.

In many crystal structures, there are internal degrees of freedom in the unit cell, as it contains more than one atom. In some cases, such as the diamond structure, symmetry arguments fix these internal degrees of freedom. In other cases relaxations within the unit cell must explicitly be done in the local region. This is an extension of the normal quasicontinuum method, but no particular difficulties are expected. The method presented here for eliminating the ghost forces can probably be extended to these cases if the extrapolation is done on the lattice vectors instead of on the interatomic distances. This would make it possible to simulate materials in the diamond structure. This has, however, not been tested yet.

\section{APPLICATIONS}

Before starting a simulation, the regions where atomic deformation gradients are expected to be small are divided into elements by a Delaunay triangulation. At the same time, $\omega_{\beta}^{e}$ is calculated. Neighbor lists for nonlocal atoms are prepared, and it is checked that there are no nonlocal atoms interacting with local atoms. For each subsequent calculation of the energy and forces, each quasi-nonlocal atom must extrapolate the neighbors it needs by using its nearest-neighbor atoms. If a quasi-nonlocal atom interacts with nonlocal atoms, then we do not need to extrapolate neighbor atoms corresponding to those atoms. After the above processes 
TABLE I. Properties of each model. In all cases, the total number of atoms is 4608 , and the size of the unit cell is $34.36 \times 39.66$ $\times 56.11 \AA^{3}$, and the crystal orientations along the $x, y$, and $z$ axes are $[\overline{1} 10],[11 \overline{2}]$, and [111], respectively.

\begin{tabular}{lcccc}
\hline \hline Model & $A$ & $B$ & $C$ & $D$ \\
\hline No. of representative atoms & 4608 & 2736 & 3408 & 3036 \\
No. of nonlocal atoms & 4608 & 1728 & 1728 & 365 \\
No. of local atoms & 0 & 1008 & 912 & 1599 \\
No. of quasi-nonlocal atoms & 0 & 0 & 768 & 1072 \\
No. of elements & 0 & 7104 & 6528 & 11681 \\
\hline \hline
\end{tabular}

have finished, we can calculate the potential energy and force of each atom. In this study, to get an equilibrium state of a system, the conjugate gradient method ${ }^{18}$ is used to define a search direction and the golden section method ${ }^{18}$ is used to find the minimum energy in that direction.

\section{A. Vacancy formation energy (point defect)}

In this section, the atomic forces in a perfect fcc crystal as well as the vacancy formation energy are calculated for aluminum using an EAM potential ${ }^{12}$ and using four different models. The cutoff distance of the potential is $6.29 \AA$ which includes fourth-nearest-neighbor atoms. Figure 6 shows schematically the atomic configurations at the center plane which is perpendicular to the $x$ direction. White, dark gray, and light gray regions in the figures, correspond to local atomic region $(L)$, nonlocal atomic region $(N)$, and quasinonlocal atomic region $(Q)$, respectively, and open circles, solid circles, and double circles indicate nonlocal atoms, local atoms, and quasi-nonlocal atoms, respectively. Periodic boundary conditions are adopted in all directions. A full atomistic simulation is performed in model $A$, an old style QC simulation with ghost forces is used in model $B$, and the improved QC method with quasi-nonlocal atoms is used in models $C$ and $D$. The important numbers for each setup are listed in Table I. The number of representative atoms is the total number of atoms in each model represented by local, nonlocal, and quasi-nonlocal atoms.

First, we calculate the potential energy and forces for the perfect fcc structure using our four models. Figure 7 shows $z$ components of the force of each model in its initial configurations. Ideally, no atomic forces should appear in the perfect fcc structure. As expected, a full nonlocal simulation (model A) gives no forces. On the other hand, nonzero forces (ghost forces) appear near the interface between nonlocal and local atomic regions in model $B$. In the models $C$ and $D$ with quasi-nonlocal atoms no ghost forces are found.

Figure 8 shows atomic displacement in the $z$ direction of the equilibrium positions from the initial perfect fcc configurations. Nonzero displacements can be observed at the interface between local and nonlocal regions in model $B$ because of the ghost forces. The relaxation of model $B$ reduces the total energy by $1.2 \mathrm{eV}$, which corresponds to a negative interface energy of $7.1 \mathrm{~mJ} / \mathrm{m}^{2}$ for a nonlocal/local interface in the (111) plane. The absolute value of the ghost plane energy corresponds to $9.3 \%$ of a $\Sigma 3$ boundary (twin boundary) energy for $\mathrm{Al}$ with the same interatomic potential.

Now we remove one atom from the center of each model in order to calculate the vacancy formation energy. In the initial configurations of models $C$ and $D$, almost the same atomic forces as those in model $A$ are obtained. Figure 9 shows atomic configurations of a $\{111\}$ plane with the vacancy in an equilibrium state for models $A, B$, and $C$. The colors correspond to the absolute value of atomic displacement (a) from the initial configuration for model $A, \mid \mathbf{r}^{\mathrm{A}}$ $-\mathbf{r}_{0}^{\mathrm{A}} \mid$ and (b) from the relaxed atomic configuration of model $A$ for model $B,\left|\mathbf{r}^{\mathrm{B}}-\mathbf{r}^{\mathrm{A}}\right|$ and for model $C,\left|\mathbf{r}^{\mathrm{C}}-\mathbf{r}^{\mathrm{A}}\right|$. As shown in Fig. 9(a), atomic displacements are distributed around the vacancy in the full atomistic simulation.

In the QC simulation with ghost forces, atomic configurations near the interface between nonlocal and local regions are different from the same region of model $A$ though atomic configurations near the vacancy are not so different from that of model $A$. The value of $\left|\mathbf{x}^{\mathrm{B}}-\mathbf{x}^{\mathrm{A}}\right|$ near the vacancy is about

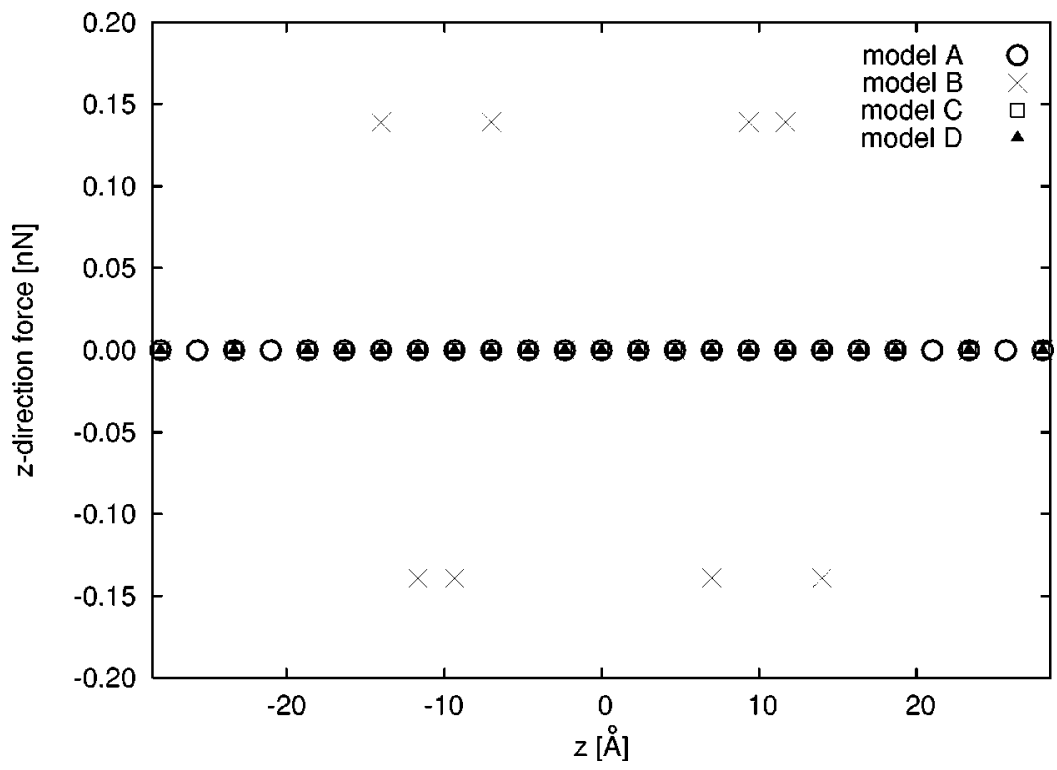

FIG. 7. Atomic force in perfect fcc structure in the initial configuration. No ghost force appears in models $C$ and $D$ with quasi-nonlocal atoms. 


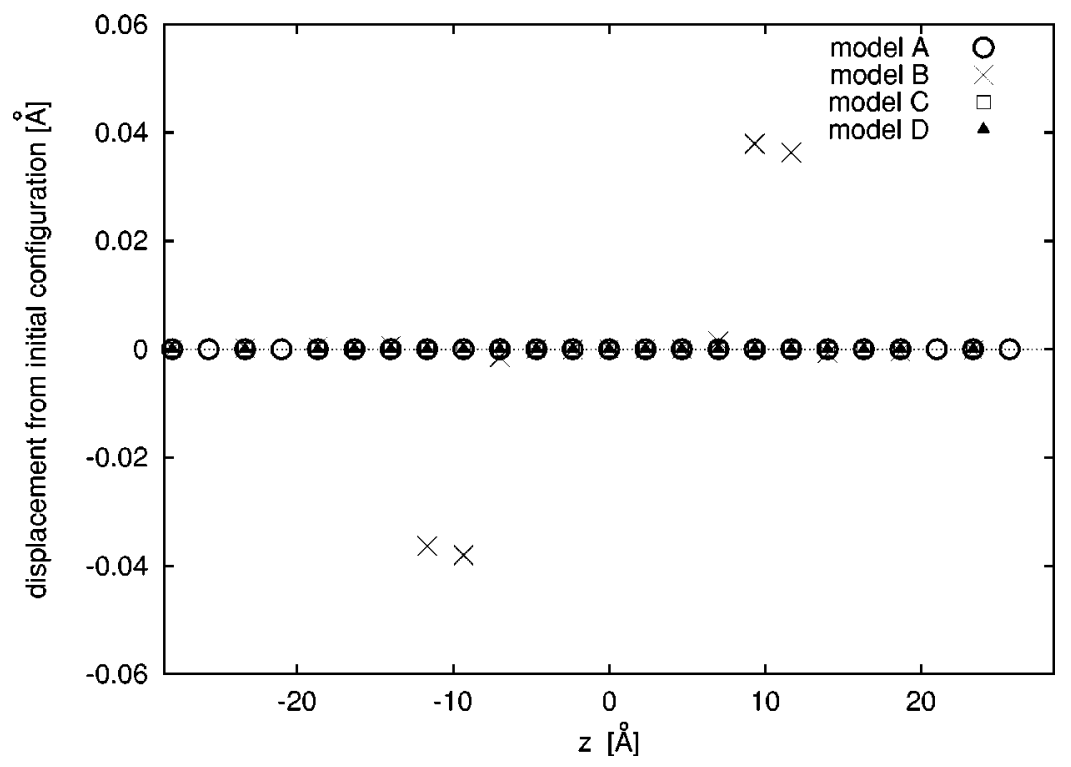

FIG. 8. Displacement in the $z$ direction of each atom from its initial configuration in the equilibrium state (perfect fcc structure).

$0.0005 \AA$. In the QC simulation with quasi-nonlocal atoms, almost exactly the same atomic displacements are obtained and it can be confirmed that the distribution of atomic displacements near the interfaces between nonlocal and quasinonlocal atoms and between quasi-nonlocal and local atoms show a truly seamless matching [see Fig. 9(b)]. The maximum value of $\left|\mathbf{x}^{\mathrm{B}}-\mathbf{x}^{\mathrm{A}}\right|$ is about $0.04 \AA$, which appears at the interface between nonlocal and local regions, and the maximum value of $\left|\mathbf{x}^{\mathrm{C}}-\mathbf{x}^{\mathrm{A}}\right|$ is about $0.0001 \AA$, which appears near the interface between quasi-nonlocal and local regions. Consequently, the same vacancy formation energy as found by full atomic simulation can be calculated by using the improved quasicontinuum method. The vacancy formation energy is $0.67 \mathrm{eV}$.

We also calculate the vacancy formation energy for $\alpha$ iron in body-centered cubic using a simple empirical $N$-body potential by Finnis and Sinclair ${ }^{13,14}$ and the same procedure as for aluminum. The cutoff distance of the potential is between
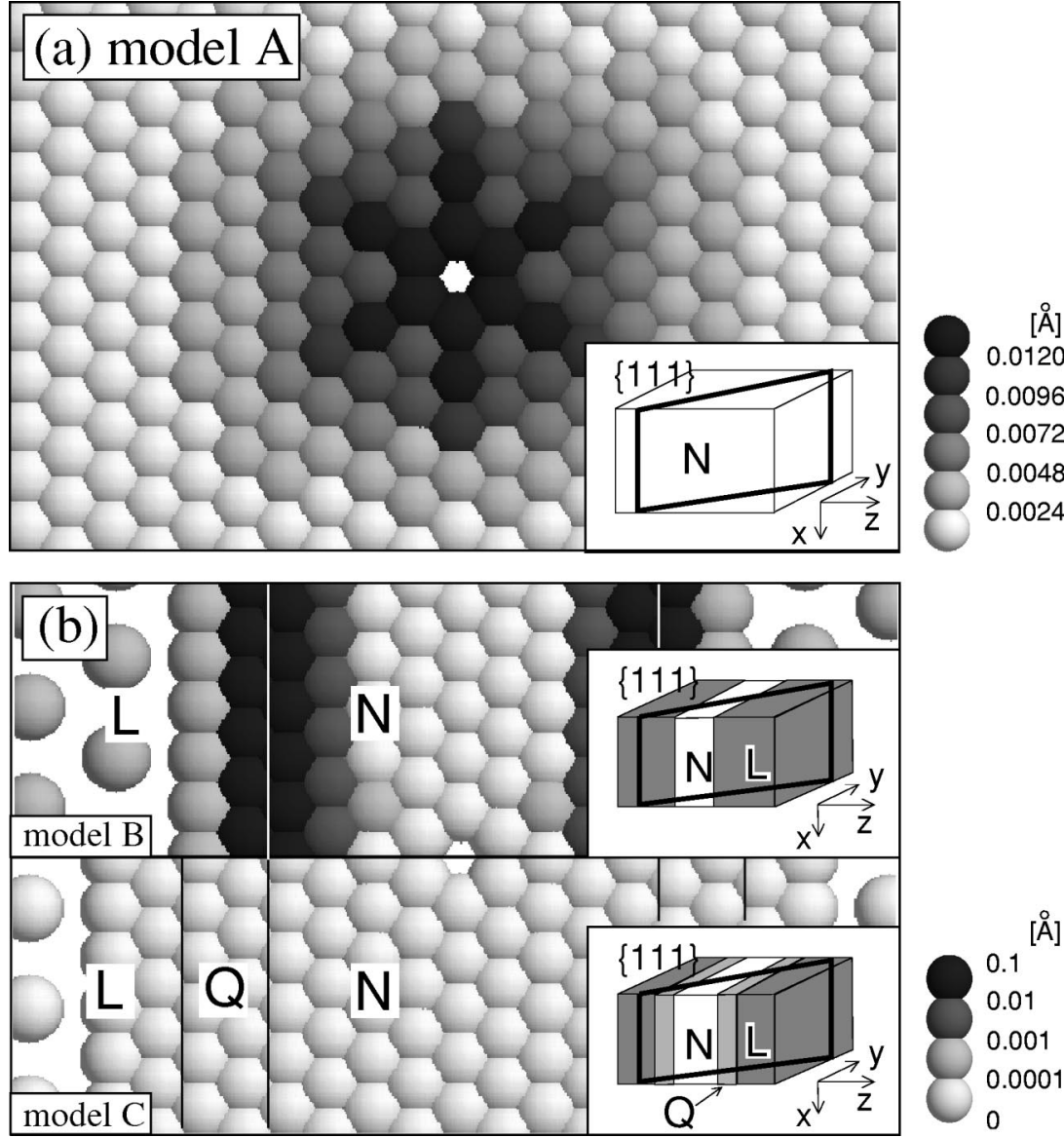

FIG. 9. Atomic configurations around the vacancy in an equilibrium state. (a) Absolute value of atomic displacement in an equilibrium state from initial configuration of model $A,\left|\mathbf{r}^{\mathrm{A}}-\mathbf{r}_{0}^{\mathrm{A}}\right|$. (b) The error in the atomic displacement in the equilibrium states of models $B$ and $C$ compared to the fully atomistic model $A:\left|\mathbf{r}^{\mathrm{B}}-\mathbf{r}^{\mathrm{A}}\right|$, and $\left|\mathbf{r}^{\mathrm{C}}-\mathbf{r}^{\mathrm{A}}\right|$. Orientation of the atomic plane is $\{111\}$. Darker colors correspond to larger atomic displacements. $\operatorname{Max}\left|\mathbf{r}^{\mathrm{B}}-\mathbf{r}^{\mathrm{A}}\right| \simeq 0.04 \AA$ and $\max \left|\mathbf{r}^{\mathrm{C}}-\mathbf{r}^{\mathrm{A}}\right| \simeq 0.0001 \AA$. 


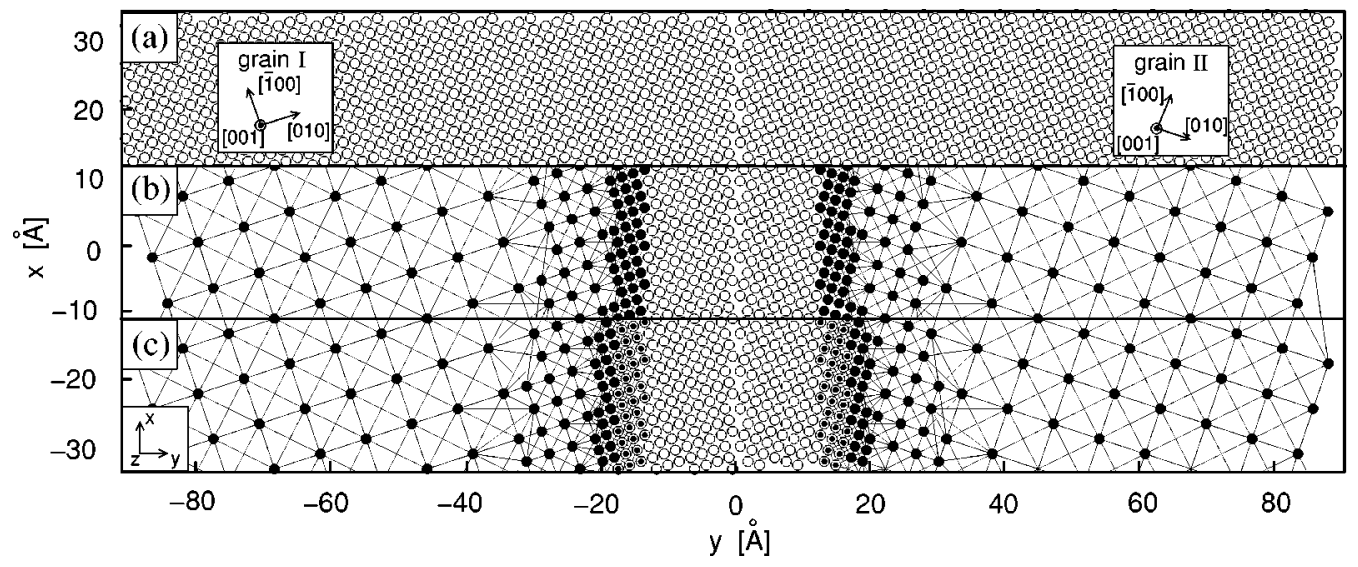

FIG. 10. A one-third part of the atomic configuration of a $\Sigma 5$ grain boundary of a full atomic model (upper part), a QC model with ghost force (middle part), and a QC model with quasi-nonlocal atoms (lower part). Open circles, solid circles, and double circles show nonlocal atoms, local atoms, and quasi-nonlocal atoms, respectively. Periodic boundary conditions are adopted in the $x$ and $z$ directions.

second- and third-neighbor shells, so it satisfies the extrapolation condition for the bcc structure. We have confirmed that in the quasicontinuum method, a seamless coupling between the coarse-grained and fully atomistic region is also obtained in the bcc structure by introducing quasi-nonlocal atoms. The vacancy formation energy is $1.83 \mathrm{eV}$.

\section{B. Grain boundary energy (plane defect)}

In this section, the energy of a $\mathrm{Cu} \Sigma 5\langle 100\rangle$ tilt grain boundary is calculated. We use an EMT potential with a cutoff distance between third- and fourth-nearest neighbors. ${ }^{15}$ Figure 10 shows one-third of the initial atomic configurations of three models: The upper part shows a full atomistic model (model $A$ ), the middle part shows a QC model with ghost force (model $B$ ), and the lower part shows our improved QC model (model $C$ ). Periodic boundary conditions are adopted in the $x$ and $z$ directions. Two initial lattice basis vectors are prepared for grains I and II, respectively. The numbers for the three models are given in Table II. The number of degrees of freedom in the QC models is a quarter of that in the full atomistic model.

Figure 11 shows atomic displacements in the $y$ direction in each equilibrium state from the initial configuration of each model. Open circles represent atomic displacement of the full atomistic model. Solid triangles and open squares represent atomic displacements in the QC model with ghost

TABLE II. Properties of each analysis model for $\Sigma 5$ grain boundary for $\mathrm{Cu}$. The dimensions of the simulation cell are 68.18 $\times 181.82 \times 57.40 \AA^{3}$.

\begin{tabular}{lccc}
\hline \hline Model & $A$ & $B$ & $C$ \\
\hline No. of representative atoms & 60864 & 13512 & 15024 \\
No. of non-local atoms & 60864 & 8448 & 8448 \\
No. of local atoms & 0 & 5064 & 3504 \\
No. of quasi-nonlocal atoms & 0 & 0 & 3072 \\
No. of elements & 0 & 35482 & 26108 \\
\hline \hline
\end{tabular}

force and the QC model with quasi-nonlocal atoms, respectively. Atomic displacements near the grain boundary for each model are nearly identical. In this region, the differences between the relaxed atomic configurations for model $A$ and model $B$ or model $C$ are about $0.0005 \AA$. However, in the interface region between local and nonlocal atoms, larger discrepancies appear in model $B$, where the error in the displacement is up to $0.02 \AA$. In model $C$ the interface is seamless, and no error is seen (the maximal deviation is $0.001 \AA$ in both the local and quasi-nonlocal regions, this is probably due to the discretization of the local region as no extra deviation is seen near the interface).

The grain boundary energy is found to be $977.5 \mathrm{~mJ} / \mathrm{m}^{2}$ for the full atomistic model and $977.4 \mathrm{~mJ} / \mathrm{m}^{2}$ for the new QC model.

\section{CONCLUSIONS}

A version of the quasicontinuum method for simple crystal structures has been formulated which avoids the problem

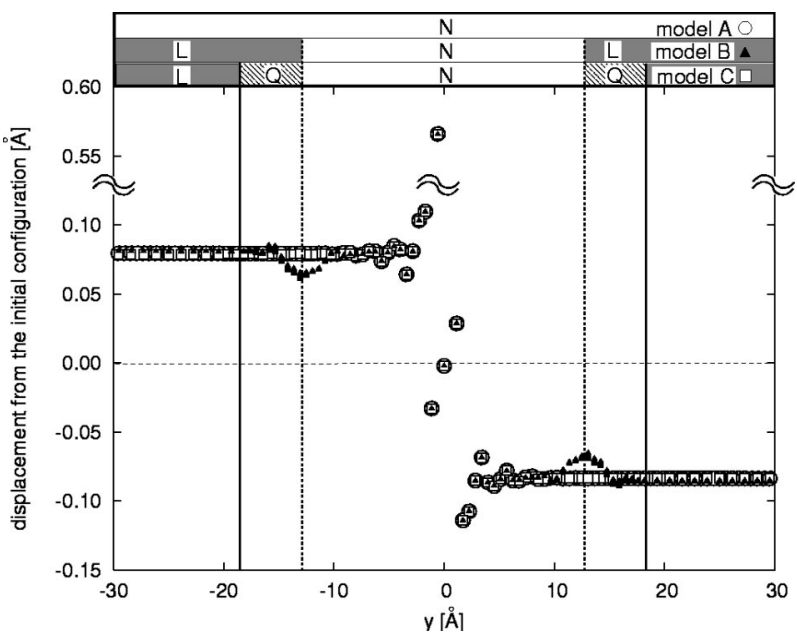

FIG. 11. Displacement from the initial configuration in an equilibrium state in the $y$ direction of the full atomic model and the QC models. $N, Q$, and $L$ are the nonlocal, quasi-nonlocal, and local atomic regions. 
of ghost forces, i.e., unphysical forces arising in the interface region between the fully atomistic (or nonlocal) region and the coarse-grained (or local) region. This is done by inserting atoms, called quasi-nonlocal atoms, between the two regions. Quasi-nonlocal atoms behave like local atoms when they interact with atoms in the local region, and as nonlocal atoms when they interact with atoms in the nonlocal region. This ensures a consistent description, free of ghost forces. As the forces obtained are conservative, there are no problems with energy conservation in dynamic simulations. In previously published methods for treating the ghost forces, the lack of energy conservation has prevented dynamic simulations. The price of eliminating the ghost forces is a slight increase in the number of degrees of freedom in the simulations, as a few extra layers of fully resolved atoms must be added to the simulation.

The quasi-nonlocal atoms use the distances to their nearest-neighbor atoms to extrapolate the positions of atoms further away, except if these positions are occupied by nonlocal atoms, in which case the actual position of the nonlocal atoms are used. It is required that the extrapolated position of a neighboring atom is obtained from displacement vectors to nearest-neighbor atoms which are also nearest-neighbor atoms of the extrapolated atom. This limits the method to interatomic potentials with sufficiently short range. The limit is fourth-, fourth-, and third-nearest neighbors for hcp, fcc, and bcc lattices, respectively.

\section{ACKNOWLEDGMENTS}

The authors gratefully acknowledge financial support from Japan Society for the Promotion of Science (JSPS) (T.S.), and from the Materials Research Program of the Danish Research Agency through Grant No. 5020-00-0012 (J.J.M., J.S., and K.W.J.). Supercomputer time has been sponsored by the Danish Center for Scientific Computing. The Center for Atomic-scale Materials Physics is sponsored by the Danish National Research Foundation.

\section{APPENDIX}

In the following we show that no ghost forces are present in the interface region, i.e., that the forces on all atoms are zero if the atoms are in a homogeneously strained fcc lattice.

The interface between the local and quasi-nonlocal atoms is trivial. The energy of a quasi-nonlocal atom near the interface (see e.g., Fig. 3) is not affected by the presence of the local atoms, since the energy of the quasi-nonlocal atom is only a function of the position of its nearest neighbors. Similarly, the energy of a local atom near the interface is not influenced by the interface, as it is just given by suitable fractions of the energies of the elements having the local atom as a corner atom.

The interface between the quasi-nonlocal atoms and the nonlocal atoms is less obvious. If all the neighbors within the cutoff distance of a quasi-nonlocal atom (for example, atom 1 in Fig. 4) are themselves quasi-nonlocal atoms, and if they are arranged in a regular lattice, there is clearly no force on the atom. If one of the neighbors, as is shown in Fig. 4(b), is replaced with a nonlocal atom, the expression for the energy of the quasi-nonlocal atom under consideration changes. If the derivative of the energy of the quasi-nonlocal atom (atom 1) with respect to its position is nonzero, this will contribute to a ghost force. However, the replacement also modifies the expression for the energy of some of the other neighboring atoms, and this also gives a contribution to the ghost force. We will show that these contributions cancel. We thus prove that there is no ghost force on quasi-nonlocal atoms near the interface to the nonlocal atoms by showing that replacing a quasi-nonlocal neighbor of an atom with a nonlocal atom does not modify the force.

In the following we calculate the change $\Delta \mathbf{F}_{1}$ of the force on atom 1 in Fig. 4(a) when one of the neighboring atoms (called atom $i$ ) is replaced with a nonlocal atom. Although the calculation is done for the two-dimensional case of Fig. 4(a), the result is easily extrapolated to three dimensions. The following notation is used: $E^{\prime}$ is the energy of the system when all neighbors are quasi-nonlocal atoms, $E$ is the energy when the neighbor $i$ is a nonlocal atom. Similarly, $E_{j}^{\prime}$ and $E_{j}$ are the energies of atom $j$ in the two configurations.

If the replaced atom $i$ is not a nearest-neighbor atom to atom 1 (e.g., $i=14$ or 15), only the atoms 1 and $i$ contribute to the change in $\mathbf{F}_{1}$ :

$$
-\Delta \mathbf{F}_{1}=\frac{\partial E^{\prime}}{\partial \mathbf{r}_{1}}-\frac{\partial E}{\partial \mathbf{r}_{1}}=\frac{\partial E_{1}^{\prime}}{\partial \mathbf{r}_{1}}-\frac{\partial E_{1}}{\partial \mathbf{r}_{1}}+\frac{\partial E_{i}^{\prime}}{\partial \mathbf{r}_{1}}-\frac{\partial E_{i}}{\partial \mathbf{r}_{1}}
$$

As atom 1 is not a nearest neighbor of atom $i$, the third term is identically zero. The term $\partial E_{1}^{\prime} / \partial \mathbf{r}_{1}$ contains two terms, one from the virtual displacement of atom 1, another from the virtual displacement of the extrapolated neighbor $i$ moving due to the displacement of atom 1:

$$
\frac{\partial E_{1}^{\prime}}{\partial \mathbf{r}_{1}}=\frac{\partial E_{1}}{\partial \mathbf{r}_{i}} \cdot \frac{\partial \mathbf{r}_{i}^{(1)}}{\partial \mathbf{r}_{1}}+\frac{\partial E_{1}}{\partial \mathbf{r}_{1}}=-\frac{\partial E_{1}}{\partial \mathbf{r}_{i}}+\frac{\partial E_{1}}{\partial \mathbf{r}_{1}}
$$

where $\mathbf{r}_{i}^{(1)}$ signifies the position of atom $i$ as seen from atom 1 , i.e., extrapolated from the neighbors of atom 1 . The quotient $\partial \mathbf{r}_{i}^{(1)} / \partial \mathbf{r}_{1}$ gives how far the extrapolated atom moves when atom 1 is moved. When atom $i$ is a second- or thirdnearest neighbor to atom 1 , it is clearly equal to -1 . In three dimensions, it is equal to -1 if atom $i$ is a second- to fourthnearest neighbor, as can be seen from Eq. (8) to Eq. (10). If interactions go beyond fourth-nearest neighbor the quotient changes from -1 , and ghost forces appear.

It has also been used that the functional form of $E_{1}^{\prime}$ and $E_{1}$ are identical when $E_{1}^{\prime}$ is written as a function of the position of all the neighbor positions and not just of the independent positions of the nearest neighbors. Inserting this in equation (A1) gives

$$
\begin{aligned}
-\Delta \mathbf{F}_{1} & =-\frac{\partial E_{1}}{\partial \mathbf{r}_{i}}+\frac{\partial E_{1}}{\partial \mathbf{r}_{1}}-\frac{\partial E_{1}}{\partial \mathbf{r}_{1}}-\frac{\partial E_{i}}{\partial \mathbf{r}_{1}} \\
& =-\left(\frac{\partial E_{1}}{\partial \mathbf{r}_{i}}+\frac{\partial E_{i}}{\partial \mathbf{r}_{1}}\right)=0,
\end{aligned}
$$

where the last parenthesis is zero for reasons of symmetry.

Proving that $\Delta \mathbf{F}_{1}$ is zero when a nearest-neighbor quasinonlocal atom is replaced with a nonlocal atom follows the 
same path, but is a little more complicated as the replacement changes $\partial E_{j} / \partial \mathbf{r}_{1}$ for all atoms $j$ which extrapolate the position of the replaced atom using the position of atom 1: If atom 6 is replaced with a nonlocal atom, then atoms 2, 3, 4, and 6 contribute to the change in the force on atom 1 (the expression for the energy of atoms 1,5 , and 7 do not change, but atoms 2, 3, and 4 use the real position of atom 6 instead of the extrapolated one). In the following, $i$ refers to the atom being replaced (6 in the example), and $A_{i}$ is the set of atoms extrapolating the position of atom $i$ using atom $1\left(A_{6}\right.$ $=\{2,3,4\})$; this is identical to the set of neighbors of atom $i$ which have their positions extrapolated using the position of atom 1:

$$
-\Delta \mathbf{F}_{1}=\frac{\partial E^{\prime}}{\partial \mathbf{r}_{1}}-\frac{\partial E}{\partial \mathbf{r}_{1}}=\frac{\partial E_{i}^{\prime}}{\partial \mathbf{r}_{1}}-\frac{\partial E_{i}}{\partial \mathbf{r}_{1}}+\sum_{j \in A_{i}}\left(\frac{\partial E_{j}^{\prime}}{\partial \mathbf{r}_{1}}-\frac{\partial E_{j}}{\partial \mathbf{r}_{1}}\right) .
$$

The first term is expanded similar to Eq. (A2), except that more terms appear as the energy $E_{i}^{\prime}$ depends on $\mathbf{r}_{1}$ through the extrapolated positions of all atoms in $A_{i}$. The terms $\partial E_{j}^{\prime} / \partial \mathbf{r}_{1}$ are also extrapolated in the same way as in Eq. (A2). This gives

$$
\begin{aligned}
-\Delta \mathbf{F}_{1}= & \frac{\partial E_{i}}{\partial \mathbf{r}_{1}}+\sum_{j \in A_{i}} \frac{\partial E_{i}}{\partial \mathbf{r}_{j}} \cdot \frac{\partial \mathbf{r}_{j}^{(i)}}{\partial \mathbf{r}_{1}}-\frac{\partial E_{i}}{\partial \mathbf{r}_{1}} \\
& +\sum_{j \in A_{i}}\left(\frac{\partial E_{j}}{\partial \mathbf{r}_{1}}+\frac{\partial E_{j}}{\partial \mathbf{r}_{i}} \cdot \frac{\partial \mathbf{r}_{i}^{(j)}}{\partial \mathbf{r}_{1}}-\frac{\partial E_{j}}{\partial \mathbf{r}_{1}}\right) \\
= & \sum_{j \in A_{i}}\left(\frac{\partial E_{i}}{\partial \mathbf{r}_{j}} \cdot \frac{\partial \mathbf{r}_{j}^{(i)}}{\partial \mathbf{r}_{1}}+\frac{\partial E_{j}}{\partial \mathbf{r}_{i}} \cdot \frac{\partial \mathbf{r}_{i}^{(j)}}{\partial \mathbf{r}_{1}}\right)=0 .
\end{aligned}
$$

In the last sum each term vanishes as $\partial \mathbf{r}_{j}^{(i)} / \partial \mathbf{r}_{1}=\partial \mathbf{r}_{i}^{(j)} / \partial \mathbf{r}_{1}$, i.e., when atom 1 moves atom 4 sees atom 6 being displaced as much as atom 6 sees atom 4 being displaced.
${ }^{1}$ A. Nakano, M. E. Bachlechner, R. K. Kalia, E. Lidorikis, P. Vashishta, G. Z. Voyiadjis, T. J. Campbell, S. Ogata, and F. Shimojo, Comput. Sci. Eng. 3 (4), 56 (2001).

${ }^{2}$ J. Q. Broughton, F. F. Abraham, N. Bernstein, and E. Kaxiras, Phys. Rev. B 60, 2391 (1999).

${ }^{3}$ R. E. Rudd and J. Q. Broughton, Phys. Rev. B 58, R5893 (1998).

${ }^{4}$ R. E. Rudd and J. Q. Broughton, Phys. Status Solidi B 217, 251 (2000).

${ }^{5}$ E. B. Tadmor, M. Ortiz, and R. Phillips, Philos. Mag. A 73, 1529 (1996).

${ }^{6}$ V. B. Shenoy, R. Miller, E. B. Tadmor, D. Rodney, R. Phillips, and M. Ortiz, J. Mech. Phys. Solids 47, 611 (1999).

${ }^{7}$ R. Miller, E. B. Tadmor, R. Phillips, and M. Ortiz, Modell. Simul. Mater. Sci. Eng. 6, 607 (1998).

${ }^{8}$ J. Knap and M. Ortiz, J. Mech. Phys. Solids 49, 1899 (2001).

${ }^{9}$ D. Rodney and R. Phillips, Phys. Rev. Lett. 82, 1704 (1999).

${ }^{10}$ R. C. Picu, J. Comput.-Aided Mater. Des. 7, 77 (2000).

${ }^{11}$ J. J. Mortensen, J. Schiøtz, and K. W. Jacobsen, SIMU Newsl. 4, 119 (2002), http://simu.ulb.ac.be/

${ }^{12}$ Y. Mishin, D. Farkas, M. J. Mehl, and D. A. Papaconstantopou- los, Phys. Rev. B 59, 3393 (1999).

${ }^{13}$ M. W. Finnis and J. E. Sinclair, Philos. Mag. A 50, 45 (1984).

${ }^{14}$ M. W. Finnis and J. E. Sinclair, Philos. Mag. A 53, 161 (1986).

${ }^{15}$ K. W. Jacobsen, P. Stoltze, and J. K. Nørskov, Surf. Sci. 366, 394 (1996).

${ }^{16}$ A. Okabe, B. Boots, and K. Sugihara, Spatial Tesselations: Concepts and Applications of Voronoi Diagrams (Wiley, Chichester, 1992).

${ }^{17}$ If a nonlocal atom is within one third of a nearest-neighbor distance from an extrapolated position, the nonlocal atom is assumed to be identical to the extrapolated one, and is therefore used. No nonlocal atoms are expected between one and two thirds of a nearest-neighbor distance from an extrapolated position, if one is found anyway the local region is too distorted to be suitable for quasi-nonlocal atoms, and the region with nonlocal atoms should be expanded.

${ }^{18}$ W. H. Press, B. P. Flannery, S. A. Teukolsky, and W. T. Vetterling, Numerical Recipes in C (Cambridge University Press, Cambridge, 1988). 